\title{
Creatine kinase-mediated improvement of function in failing mouse hearts provides causal evidence the failing heart is energy starved
}

\author{
Ashish Gupta, ${ }^{1,2}$ Ashwin Akki, ${ }^{1,2}$ Yibin Wang, ${ }^{3}$ Michelle K. Leppo, ${ }^{1}$ V.P. Chacko, ${ }^{2}$
} D. Brian Foster, ${ }^{1}$ Viviane Caceres, ${ }^{1}$ Sa Shi, ${ }^{1,4}$ Jonathan A. Kirk, ${ }^{1}$ Jason Su, ${ }^{5}$ Shenghan Lai, ${ }^{4,5}$ Nazareno Paolocci, ${ }^{1,6}$ Charles Steenbergen, ${ }^{5}$ Gary Gerstenblith, ${ }^{1,2}$ and Robert G. Weiss ${ }^{1,2}$

${ }^{1}$ Department of Medicine, Cardiology Division, and 2Department of Radiology, Division of Magnetic Resonance Research, The Johns Hopkins University School of Medicine, Baltimore, Maryland, USA. ${ }^{3}$ Division of Molecular Medicine, Department of Anesthesiology, The Cardiovascular Research Laboratories, David Geffen School of Medicine at UCLA, Los Angeles, California, USA. ${ }^{4}$ Department of Pathophysiology, Harbin Medical University, Harbin, China. ${ }^{5}$ Department of Pathology, The Johns Hopkins University School of Medicine, Baltimore, Maryland, USA. ${ }^{6}$ Department of Clinical and Experimental Medicine, Section of General Pathology, University of Perugia, Perugia, Italy.

\begin{abstract}
ATP is required for normal cardiac contractile function, and it has long been hypothesized that reduced energy delivery contributes to the contractile dysfunction of heart failure (HF). Despite experimental and clinical HF data showing reduced metabolism through cardiac creatine kinase $(\mathrm{CK})$, the major myocardial energy reserve and temporal ATP buffer, a causal relationship between reduced ATP-CK metabolism and contractile dysfunction in HF has never been demonstrated. Here, we generated mice conditionally overexpressing the myofibrillar isoform of CK (CK-M) to test the hypothesis that augmenting impaired CK-related energy metabolism improves contractile function in HF. CK-M overexpression significantly increased ATP flux through CK ex vivo and in vivo but did not alter contractile function in normal mice. It also led to significantly increased contractile function at baseline and during adrenergic stimulation and increased survival after thoracic aortic constriction (TAC) surgery-induced HF. Withdrawal of CK-M overexpression after TAC resulted in a significant decline in contractile function as compared with animals in which CK-M overexpression was maintained. These observations provide direct evidence that the failing heart is "energy starved" as it relates to CK. In addition, these data identify $\mathrm{CK}$ as a promising therapeutic target for preventing and treating $\mathrm{HF}$ and possibly diseases involving energy-dependent dysfunction in other organs with temporally varying energy demands.
\end{abstract}

\section{Introduction}

Heart failure (HF) is typically defined as an inability of the heart to pump sufficient blood to meet the needs of the body at normal filling pressures (1) and is a major and growing public health problem throughout the world. It affects about 5 million patients in the United States alone, with approximately 550,000 new cases diagnosed each year (2). More Medicare dollars are spent for the diagnosis and treatment of HF than for any other diagnosis, and the total direct and indirect annual costs of HF in the United States have been estimated at approximately $\$ 27.9$ billion (2). Because ATP is absolutely required to fuel ongoing normal contractile function, the possibility that inadequate ATP supply underlies HF has been the foundation for the decades-old, but still unproven, hypothesis that the failing heart is "energy starved" (3-5).

The creatine kinase $(\mathrm{CK})$ reaction is the major energy reserve of the heart, providing ATP cyclically during the cardiac cycle and during periods of increased demand, as it rapidly and reversibly converts creatine phosphate $(\mathrm{PCr})$ and ADP to ATP and creatine

Conflict of interest: Under a licensing agreement with NanoCor Therapeutics Inc., Robert G. Weiss is entitled to a share of royalty on sales of technology related to creatine kinase gene therapy. Weiss is also a paid consultant to and Scientific Advisory Board member of NanoCor Therapeutics Inc. The terms of this arrangement are being managed by the Johns Hopkins University in accordance with its conflict-ofinterest policies.

Citation for this article: J Clin Invest. 2012;122(1):291-302. doi:10.1172/JCI57426.
(Cr) $(6,7)$. Abnormalities in cardiac CK metabolism are present in nearly all forms of experimental and clinical HF, including a reduction in CK reactants (e.g., PCr, Cr, and ATP), altered CK isoforms, and reduced total CK activity $(3-5,8,9)$. Despite the association between reduced CK activity and experimental HF across many species and in human HF (8-12), there has been no direct causal evidence showing that increasing depressed CK metabolism improves contractile function in the failing heart. In fact, the observation that transgenic constitutive CK-knockout mice do not develop overt HF (13) argues against a causal role. Thus, it has not been clear whether altered CK energy metabolism is simply one of many consequences of HF or whether it contributes mechanistically to the development and/or progression of contractile dysfunction in HF.

Because the loss of total CK activity potentially contributes more to reduced CK flux in experimental and human HF than do modest reductions in substrates driving the CK reaction $(14,15)$, increasing CK expression is a logical target to improve CK metabolism in the failing heart in order to test this hypothesis. We utilized conditional, transgenic overexpression techniques to augment CK activity in murine hearts and thoracic aortic constriction (TAC) to induce pressure-overload hypertrophy, contractile dysfunction, and ventricular dilatation (16), producing a model that shares many energetic, anatomic, and functional characteristics with human HF (17). We used recent magnetic resonance spectroscopy 

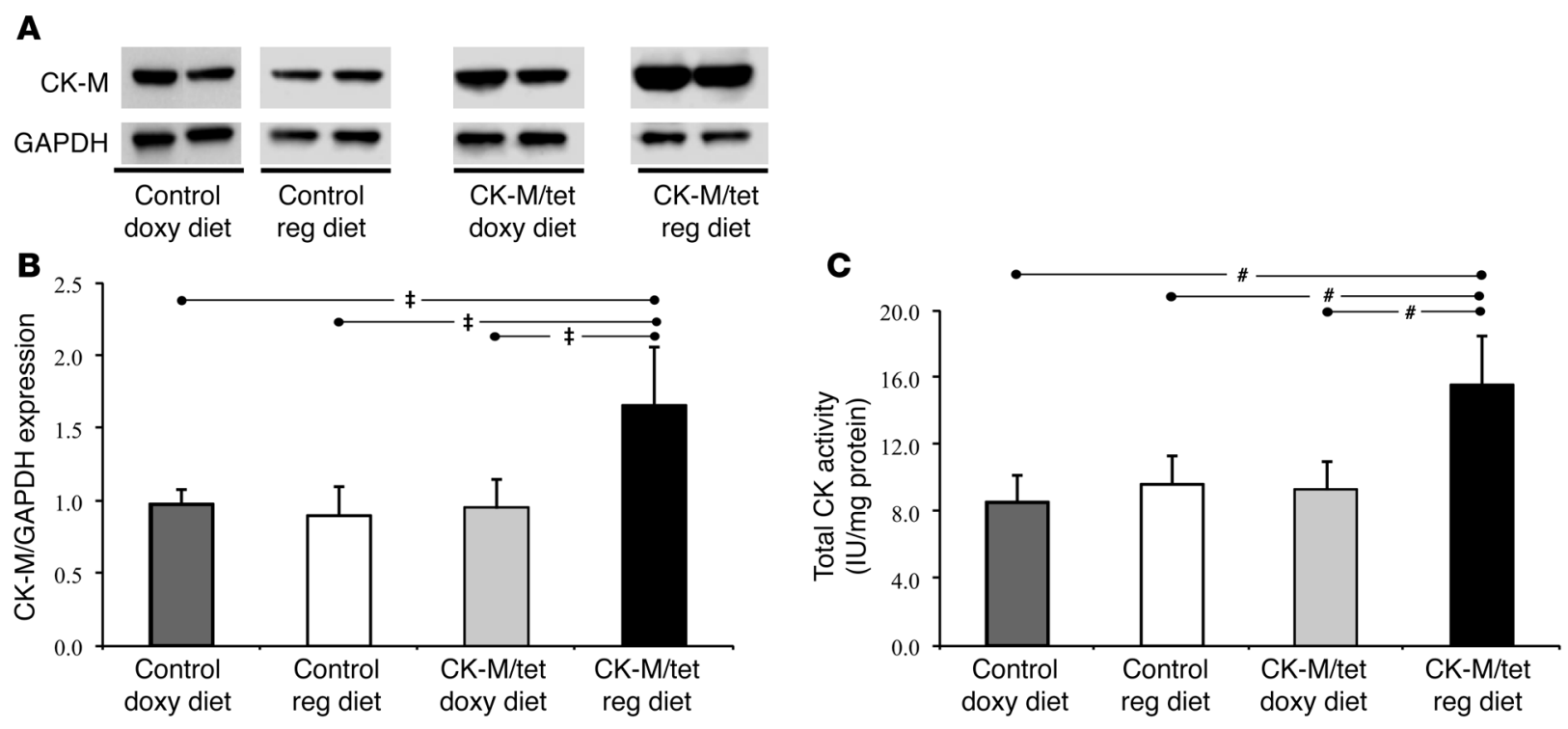

Figure 1

Effects of CK-M overexpression on protein content and CK activity. (A) Western blots and summary results, (B) CK-M protein expression, and (C) total CK activity (IU/mg protein) in control mice on a doxycycline (doxy) diet $(n=6)$, control on regular (reg) diet $(n=10)$, CK-M/tet on a doxycycline diet $(n=6)$, and CK-M/tet on regular diet $(n=7)$ mouse hearts. Results are mean $\pm \mathrm{SD}$. $\# P<0.01, \neq P<0.005$.

(MRS) and imaging (MRI) techniques adapted for rapidly beating mouse hearts $(18,19)$ to quantify the in vivo energetic and contractile consequences of the metabolic gene therapy.

\section{Results}

$C K$ overexpression and cardiac energy metabolism in the in vitro heart. Mice transgenic for CK-M and tetracycline-controlled transactivator (tTA) who were switched from a doxycycline-containing diet to a regular diet (hereafter designated CK-M overexpressors or Overexp) in this "Tet-off" model increased CK-M protein expression and in vitro total CK activity by approximately $70 \%$ over that in the control animals (Figure 1, A-C). This did not, however, significantly alter the activity of other cardiac phospho-transfer reactions such as hexokinase or adenylate kinase (Supplemental Table 1; supplemental material available online with this article; doi:10.1172/JCI57426DS1). Augmentation of CK-M, a cytosolic isoform, did not alter mitochondrial anatomy or function (Supplemental Figures 1 and 2).

With confirmation that the transgenic mice augment cardiac CK-M expression and total CK activity in response to doxycycline manipulation, isolated perfused mouse heart studies were performed to determine the effect of CK-M overexpression on myocardial highenergy phosphate metabolism under perfusion conditions where coronary flow and substrates could be identically matched between CK-M overexpressing and control mice. Hearts were isolated following sham surgery or 4 or 9 weeks after TAC surgery. TAC surgery resulted in a significant, progressive increase in heart weight that was similar in control and CK-M overexpressing animals (Table 1). TAC also caused a reduction in CK-M expression and total CK activity (Figure 2). Importantly, CK-M overexpression in TAC hearts significantly increased the depressed CK-M protein levels and CK activity above that of control TAC hearts (Figure 2). Cardiac highenergy phosphates and the rates of ATP synthesis through CK were studied with a combination of biochemical and saturation transfer magnetic resonance (MR) spectroscopy techniques (Figures 3 and 4). CK-M overexpression did not change cardiac PCr/ATP or ATP concentrations (hereafter designated [ATP]) in sham animals, although it did increase the CK pseudo-first-order rate constant and the rate of ATP synthesis through CK several-fold in isolated beating perfused hearts from sham animals (Figure $3, \mathrm{~A}$ and $\mathrm{B}$, and Figure $4 \mathrm{~A})$. In contrast, CK-M overexpression attenuated the decline in PCr/ATP and [ATP] in TAC hearts (Figure 4, B and C).

Table 1

Morphology of control and CK-M overexpressing mice

\begin{tabular}{|c|c|c|c|c|c|c|}
\hline \multirow[t]{2}{*}{ Model } & \multicolumn{2}{|c|}{ Sham } & \multicolumn{2}{|c|}{ 4-Week TAC } & \multicolumn{2}{|c|}{ 9-Week TAC } \\
\hline & $\begin{array}{l}\text { Control } \\
(n=10)\end{array}$ & $\begin{array}{l}\text { CK-M } \\
(n=9)\end{array}$ & $\begin{array}{l}\text { Control } \\
(n=7)\end{array}$ & $\begin{array}{c}\text { CK-M } \\
(n=10)\end{array}$ & $\begin{array}{l}\text { Control } \\
(n=5)\end{array}$ & $\begin{array}{c}\text { CK-M } \\
(n=7)\end{array}$ \\
\hline HW (mg) & $156 \pm 13.4$ & $147 \pm 10.9$ & $234 \pm 47.2^{\mathrm{A}}$ & $231 \pm 29.7^{B}$ & $324 \pm 64.7^{\mathrm{A}}$ & $317 \pm 26.1^{\mathrm{B}}$ \\
\hline BW (g) & $37.1 \pm 5.3$ & $37.3 \pm 4.7$ & $35.8 \pm 7.3$ & $36.8 \pm 5.6$ & $35.5 \pm 4.6$ & $32.8 \pm 8.7$ \\
\hline HW/BW (mg/g) & $4.2 \pm 0.5$ & $4.0 \pm 0.4$ & $6.6 \pm 1.3^{A}$ & $6.3 \pm 0.7^{B}$ & $9.1 \pm 1.4^{A}$ & $10.2 \pm 2.6^{B}$ \\
\hline HW/tibia (mg/mm) & $8.5 \pm 0.6$ & $8.2 \pm 0.7$ & $12.8 \pm 2.4^{\mathrm{A}}$ & $12.2 \pm 1.5^{\mathrm{B}}$ & $17.5 \pm 3.2^{A}$ & $16.7 \pm 1.4^{\mathrm{B}}$ \\
\hline
\end{tabular}

HW, heart weight; BW, body weight. ${ }^{A} P<0.05$ versus control sham. ${ }^{B} P<0.05$ versus CK-M sham. 
A

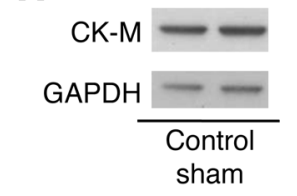

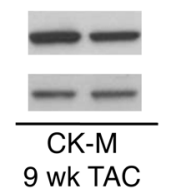

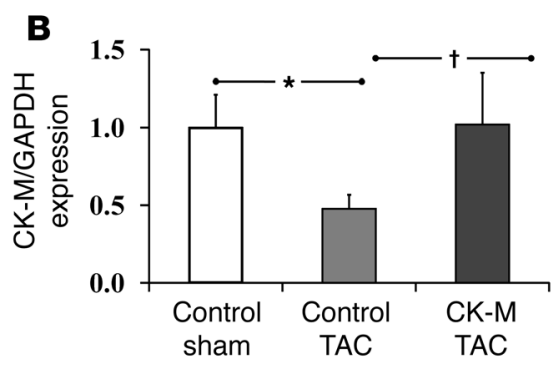

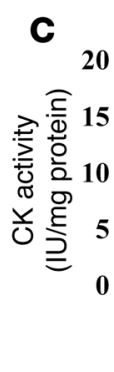

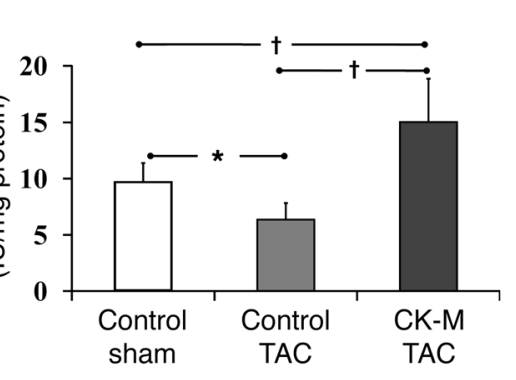

Figure 2

CK expression and activity in TAC hearts. Effect of TAC and CK-M overexpression on CK-M protein expression (A and $\mathbf{B}$ ) and CK activity (C) in control sham, control TAC, and CK-M TAC isolated mouse hearts. Results are mean $\pm \mathrm{SD}$; $n=6-17$ each group. ${ }^{*} P<0.05,{ }^{\dagger} P<0.001$.
The rate of ATP synthesis through CK in TAC hearts was significantly higher in CK-M overexpressing hearts than that in control TAC hearts and was maintained at levels comparable to those in control sham animals (Figure 4, B and C). Thus, CK-M overexpression significantly increased the rate of ATP synthesis through CK in normal and TAC failing mouse hearts, and this was associated with modest but significant increases in cardiac high-energy phosphate stores in failing TAC hearts.

In vivo metabolic, functional, and survival effects of CK-M overexpression. At baseline, there were no differences in either anatomic or functional cardiac parameters, including LV mass (113 $\pm 14 \mathrm{mg}$ vs. $112 \pm 14 \mathrm{mg}, P=\mathrm{NS}$ ), end-diastolic volume (EDV; $77 \pm 15 \mu \mathrm{l}$ vs. $71 \pm 11 \mu \mathrm{l}, P=\mathrm{NS}$ ), end-systolic volume (ESV; $27 \pm 6 \mu \mathrm{l}$ vs. $25 \pm 5 \mu \mathrm{l}$, $P=\mathrm{NS}$ ), stroke volume (SV; $50 \pm 10 \mu \mathrm{l}$ vs. $46 \pm 7 \mu \mathrm{l}, P=\mathrm{NS})$, or ejection fraction (EF; $65 \% \pm 5 \%$ vs. $64 \% \pm 1 \%, P=\mathrm{NS}$ ) in CK-M overexpressor versus control mice, respectively. There was also no difference in cardiac PCr/ATP, measured in vivo, between control and CK-M overexpressing mice (Figure 5D).

CK-M overexpression prior to TAC (Figure 5A) attenuated the decline in cardiac PCr/ATP in vivo that followed TAC surgery (Figure 5D). Specifically, mean in vivo cardiac PCr/ATP was modestly, but significantly, higher in CK-M overexpressors than in control animals at both 4 and 8 weeks after TAC (Figure 5D).

CK-M overexpression significantly improved LV systolic contractile function in TAC hearts (Figure 5, B and C). Specifically, at 4 and 8 weeks following TAC, mean $\mathrm{EF}(P<0.001), \mathrm{SV}(P<0.001)$, and cardiac output (CO; $P<0.0001)$ were significantly higher in CK-M overexpressors as compared with control animals (Figure 5C). CK-M overexpression had no effect on LV mass at either 4 weeks $(197 \pm 14 \mathrm{mg}$ vs. $203 \pm 30 \mathrm{mg}, P=\mathrm{NS})$ or 8 weeks after TAC (214 \pm 16 mg vs. $227 \pm 19$ mg, CK-M overexpressors versus control, respectively, $P=\mathrm{NS}$ ). Survival after TAC was significantly improved with CK-M overexpression (Figure 5E). Thus CK-M overexpression improves the in vivo energetic profile, significantly enhances contractile function (EF, SV, and $\mathrm{CO}$ ), and improves survival in murine TAC-induced LV dysfunction.

Reversibility of functional effects of CK-M overexpression after TAC. Because impaired energy metabolism may play varied roles during different stages of heart failure development and progression, we performed additional studies in which CK-M was reversibly overexpressed to determine whether the long-term contractile benefits, shown in the first series of experiments (Figure 5), would cease after CK-M was no longer overexpressed. In this experiment,
TAC was performed in control and CK-M-overexpressing mice, with the CK-M overexpression being discontinued later in some animals (Figure 6A).

Four weeks after TAC, mean contractile function was better in CK-M overexpressors $(n=19)$ than in control mice $(n=10$, Figure $6 \mathrm{~B})$, confirming the results of the first series of experiments (Figure 5). Specifically, EF ( $43 \% \pm 8 \%$ vs. $35 \% \pm 4 \%, P<0.01)$, SV $(45 \pm 8 \mu \mathrm{l}$ vs. $26 \pm 4 \mu \mathrm{l}, P<0.001)$, and $\mathrm{CO}(20.7 \pm 6.1 \mathrm{ml} / \mathrm{min} v \mathrm{~s} .11 .9 \pm 2.5 \mathrm{ml} / \mathrm{min}$, $P<0.001)$ were significantly higher in CK-M overexpressors than in control animals. Four weeks after TAC, CK-M overexpression was stopped in some, but not all, of the double transgenic animals by returning them to a doxycycline-containing diet (Figure 6A). In vivo contractile function was studied 8 weeks later (12 weeks after TAC). Contractile function was better in CK-M-overexpressing mice at 12 weeks than in those in which CK-M overexpression was discontinued in terms of $\mathrm{EF}(39 \% \pm 4 \%$ vs. $30 \% \pm 6 \%, P<0.05), \mathrm{SV}(59 \pm 10 \mu \mathrm{l}$ vs. $36 \pm 12 \mu \mathrm{l}, P<0.05)$, and $\mathrm{CO}(26.6 \pm 6.1 \mathrm{ml} / \mathrm{min} v \mathrm{~s} .17 .9 \pm 5.2 \mathrm{ml} / \mathrm{min}$, $P=0.07$; Figure 6B). The reduced function in CK-M withdrawal animals was not due to a negative inotropic effect of doxycycline, because contractile parameters in the doxycycline-fed control TAC animals and the control TAC animals fed a regular diet 12 weeks after TAC were similar (EF $30 \% \pm 4 \%$ vs. $34 \% \pm 5 \%, P=\mathrm{NS}$ ), SV $(30 \pm 2 \mu \mathrm{l}$ vs. $36 \pm 12 \mu \mathrm{l}, P=\mathrm{NS})$, and $\mathrm{CO}(13.8 \pm 1.1 \mathrm{ml} / \mathrm{min}$ vs. $15.2 \pm 7.3 \mathrm{ml} / \mathrm{min}$, control doxycycline $[n=6]$ vs. control-regular $\operatorname{diet}[n=7], P=\mathrm{NS}$ ). Thus, CK-M overexpression improved contractile function in TAC hearts, but this improvement did not persist after discontinuation of the CK-M transgene overexpression.

Effects of CK-M overexpression during adrenergic stress in normal and failing hearts. Because CK is the primary energy reserve of the heart and because CK inhibition limits contractile reserve (i.e., the contractile response to adrenergic stimulation) $(20,21)$, additional in vivo studies were performed to determine the extent to which CK-M overexpression augments contractile reserve in normal and failing hearts. Functional and metabolic parameters were assessed by MRI/MRS before and after the administration of dobutamine $(22,23)$ in control and CK-M overexpressor mice (Figure 7$)$. As observed in the earlier series of experiments, under baseline conditions, contractile function was similar in control and CK-M-overexpressing mice, but it was significantly improved for overexpressors after TAC (Supplemental Figures 3 and 4).

The heart rate response to dobutamine was similar in control and CK-M overexpressor animals in both sham (Figure 7A) and 4-week TAC (Figure 7B) hearts. In sham hearts, CK-M overexpres- 
A

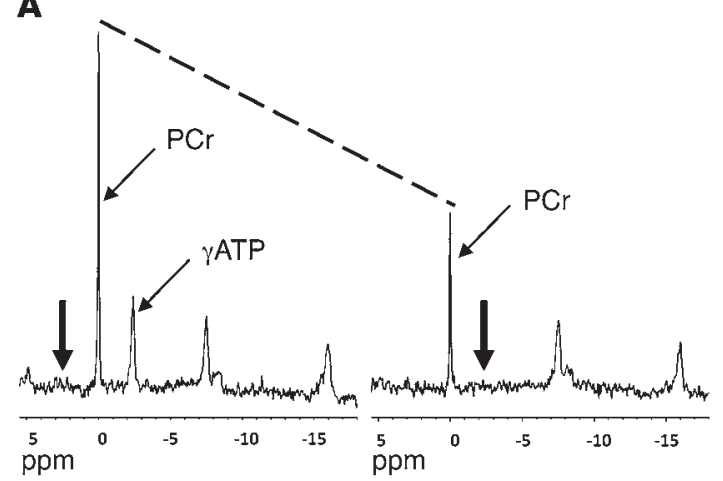

C

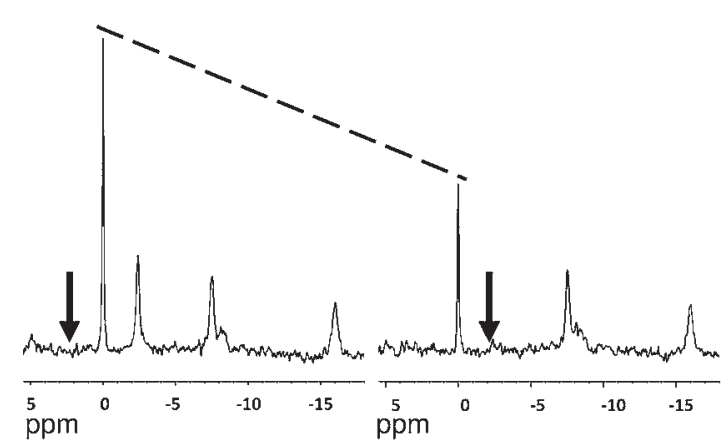

$\mathbf{E}$
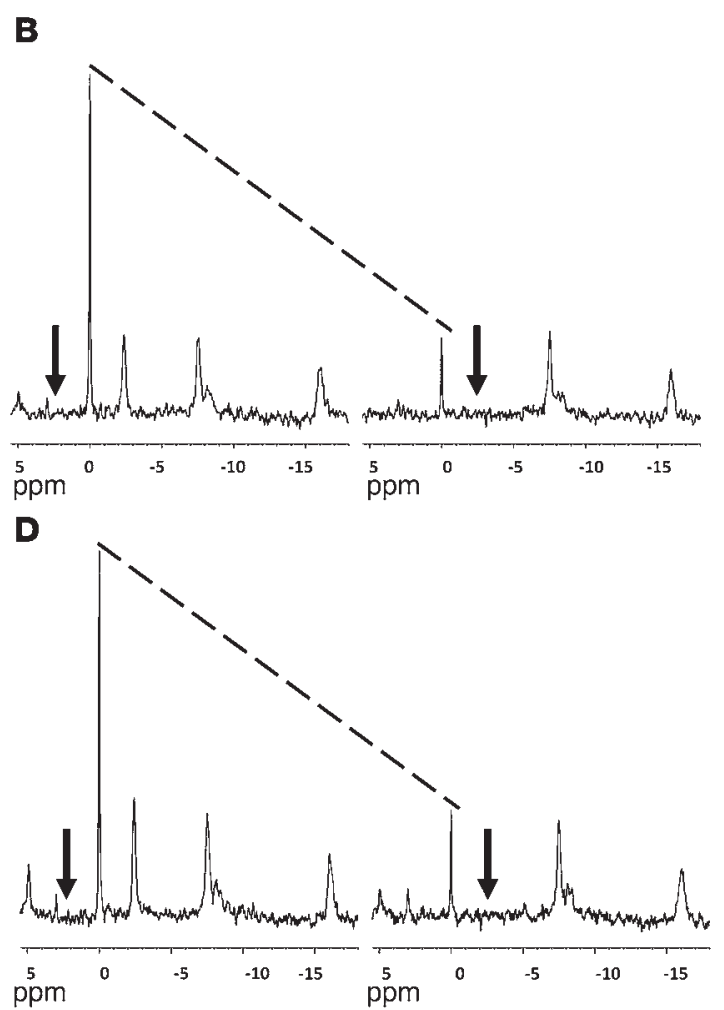

$\mathbf{F}$

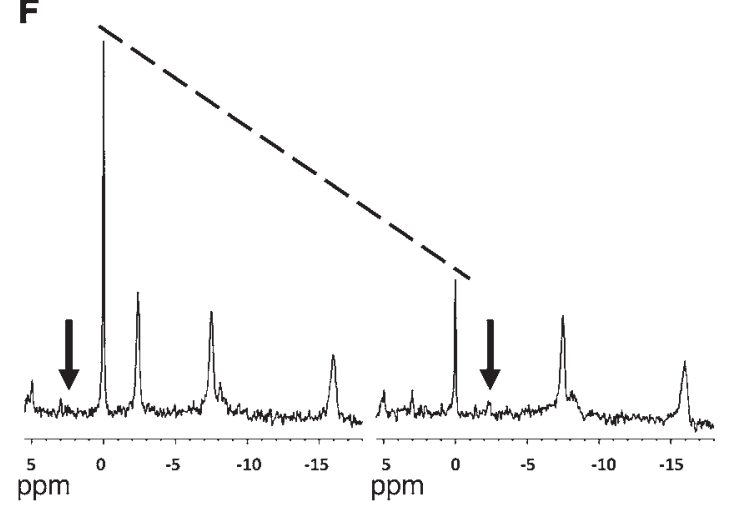

Figure 3

Perfused heart ${ }^{31} \mathrm{P}$ MR spectroscopy. Representative ${ }^{31} \mathrm{P}$ saturation transfer MR spectra are shown for $(\mathbf{A}$ and $\mathbf{B})$ sham, control, and CK-M overexpressors, respectively; (C and D) 4-week TAC, control, and CK-M overexpressors; and for (E and F) 9-week TAC, control, and CK-M overexpressors. Spectra were acquired in the presence of saturating irradiation (arrows) either in the control (left spectrum in each pair) or $\gamma$-ATP position (right spectrum). The decrease in the height of PCr peak between control and $\gamma$-ATP saturation (dotted lines) is directly related to the rate of ATP synthesis through the CK reaction.

sion did not affect the contractile response to adrenergic stimulation (Figure $7, \mathrm{C}$ and $\mathrm{E})$ or the in vivo cardiac $\mathrm{PCr} / \mathrm{ATP}$ ratio $(1.95 \pm 0.07$ vs. $1.88 \pm 0.10$, baseline vs. dobutamine, $P=\mathrm{NS}$ ). In TAC hearts, in contrast, $\mathrm{CK}-\mathrm{M}$ overexpression resulted in a greater increase in $\mathrm{EF}$ from baseline $(54 \% \pm 9 \%$ vs. $37 \% \pm 11 \%$, relative increases for CK-M vs. control, $P<0.005$, Figure 7D) and a greater decrease in ESV from baseline $(-58 \% \pm 16 \%$ vs. $-22 \% \pm 7 \%, P<0.001$; Figure $7 \mathrm{~F})$ as compared with that in control hearts. Mean CO was significantly higher in CK-M overexpressing than in control hearts during dobutamine treatment $(31.7 \pm 5.3 \mathrm{ml} / \mathrm{min}$ vs. $24.4 \pm 1.9 \mathrm{ml} / \mathrm{min}$, CK-M vs. control; $P<0.006$, Supplemental Figure 4I), but the dobutamine-induced changes in $\mathrm{CO}$ from baseline were similar in the two groups $(75 \% \pm 19 \%$ vs. $68 \% \pm 11 \%, P=\mathrm{NS})$. The myocardial $\mathrm{PCr} / \mathrm{ATP}$ ratio was higher after TAC at baseline in CK-M overexpressor versus control hearts $(1.76 \pm 0.11$ vs. $1.60 \pm 0.14, P<0.024)$, consistent with earlier experiments (Figure 4 and Figure 5D), but was unchanged during dobutamine treatment in both CK-M $(1.77 \pm 0.15, P<\mathrm{NS})$ and control $(1.59 \pm 0.17, P=\mathrm{NS})$ TAC animals. Thus, in vivo myocardial high-energy phosphates were maintained in CK-M overexpressing TAC hearts despite the higher CO and increased contractile response to adrenergic stimulation.

Effect of CK-M overexpression on in vivo ATP synthesis rates. The significantly improved contractile function of CK-M overexpressor TAC hearts was associated with a significant, but relatively mod- 
A

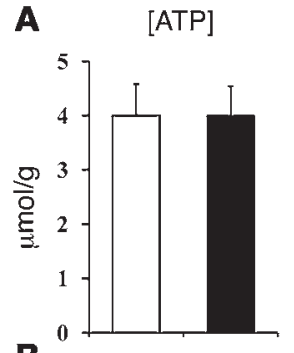

B

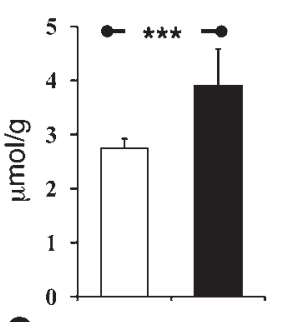

c

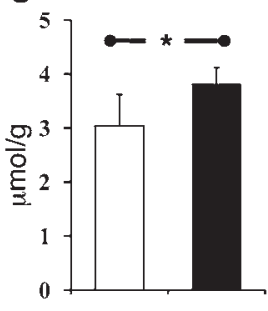

PCr/ATP
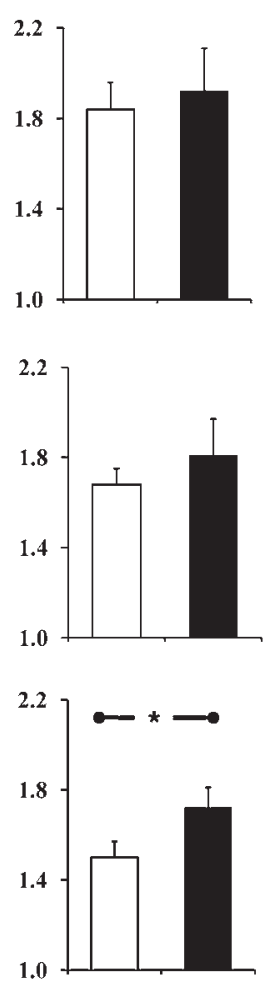

T1
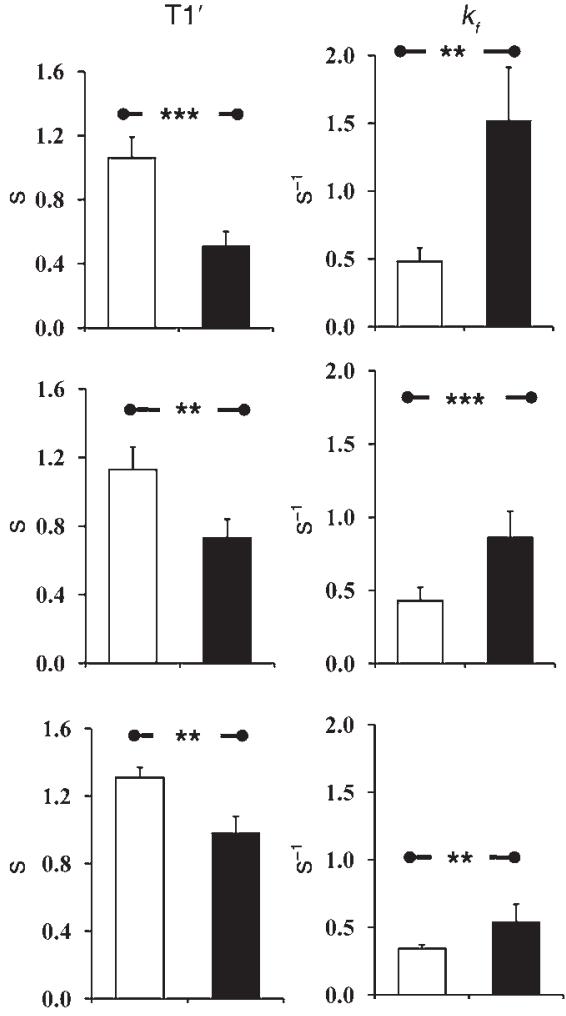
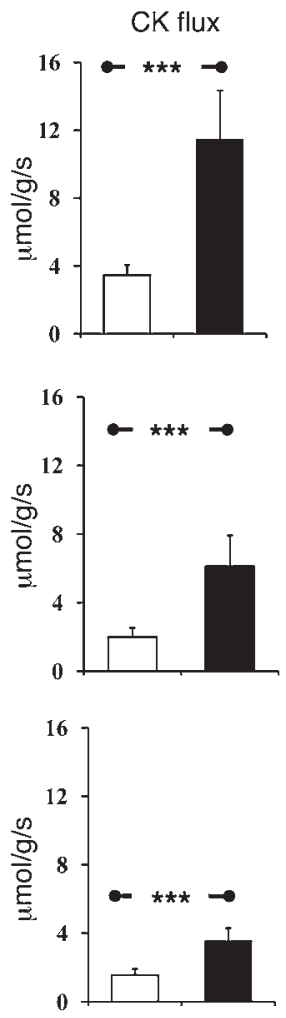

\section{Figure 4}

Summary of energetic findings in perfused hearts. ATP concentration ( $\mu \mathrm{mol} / \mathrm{g}$ of wet weight), PCr/ATP ratio, myocardial $\mathrm{T}_{1}{ }^{\prime}$ for PCr(s), $\mathrm{pseudo-first-}$ order forward rate constant, $k_{f}\left(\mathrm{~s}^{-1}\right)$, and ATP flux through CK $(\mu \mathrm{mol} / \mathrm{g} / \mathrm{s})$ in control (white bars) and CK-M overexpressing (black bars) mouse hearts in the sham (A), 4-week TAC (B), and 9-week TAC (C) groups. Results are mean \pm SD; $n=5-10$ in each group. ${ }^{\star} P<0.05,{ }^{\star \star} P<0.01,{ }^{\star * \star} P<0.001$.

est, improvement of in vivo myocardial PCr/ATP ratio (Figure 5D and Figure 8E). Based on prior studies in experimental and human hypertrophied and failing hearts showing that the rate of ATP synthesis through the $\mathrm{CK}$ reaction is more markedly reduced than are the relative and absolute high-energy phosphate pools $(12,14,15)$, we posited that CK-M overexpression likely has a greater impact on the in vivo rate of ATP synthesis through CK than on PCr/ATP. This would be consistent with our observations in isolated hearts (Figures 3 and 4) and possibly suggest that increased myofibrillar ATP delivery may account for the contractile improvement in failing CK-M hearts. We therefore used in vivo saturation transfer techniques recently adapted for the mouse heart (12) to determine the extent to which the rate of ATP synthesis is augmented in hypertrophied and failing hearts by CK-M overexpression in vivo. A representative high-resolution transverse ${ }^{1} \mathrm{H}$ MR cardiac image and corresponding spatially localized cardiac ${ }^{31} \mathrm{P}$ MR spectra under different chemically sensitive saturating conditions from the in vivo mouse heart and summary data are shown in Figure 8. The mean in vivo cardiac $k_{f}$, the pseudo-first-order rate constant, increased almost 2 -fold in CK-M-overexpressing mice (Figure $8 \mathrm{H}$ ). Critically, the in vivo rate of ATP synthesis through cardiac CK was significantly increased under non-TAC and TAC conditions in CK-M-overexpressing mice as compared with control animals, and, in fact, the rate of ATP synthesis in the CK-M-overexpressing TAC mice was similar to that in control mice that did not undergo TAC (Figure 8I). These findings are consistent with the premise that CK-M overexpression primarily enhances myocardial energy metabolism in the failing heart by enhancing the rate of myofibrillar ATP delivery rather than by only augmenting the PCr/ATP or the size of myocardial high-energy phosphate pools.

Effect of CK-M overexpression on excitation-contraction coupling in isolated myocytes. To determine whether the improvement in contractile function in CK-M-overexpressing TAC hearts was related to fundamental changes in myocyte function and/or calcium handling, we performed isolated myocyte studies. Sarcomere shortening was decreased and sarcomere relaxation time increased with TAC (Figure 9, A and B). There were no differences in sarcomere shortening or calcium transient amplitude between control and CK-M overexpressor myocytes for control or for TAC animals (Figure 9, A-D), although calcium relaxation time increased in CK-M TAC versus control TAC (Figure 9D). Taken together, these results indicate that the protective benefit of CK-M overexpression observed in vivo is not present in the single, isolated myocyte. They suggest there are no fundamental mechanical changes in the isolated, unloaded myocyte and that the in vivo contractile and survival benefits of CK-M overexpression arise primarily from the augmented myofibrillar energy delivery afforded by CK to the intact, hemodynamically loaded mouse heart.

\section{Discussion}

Although metabolic abnormalities and, in particular, reduced CKmediated metabolism have been identified in nearly all experimental HF models and indeed in human $\operatorname{HF}(3,5,24)$, it has not been clear whether reduced CK metabolism contributes to, or is only 

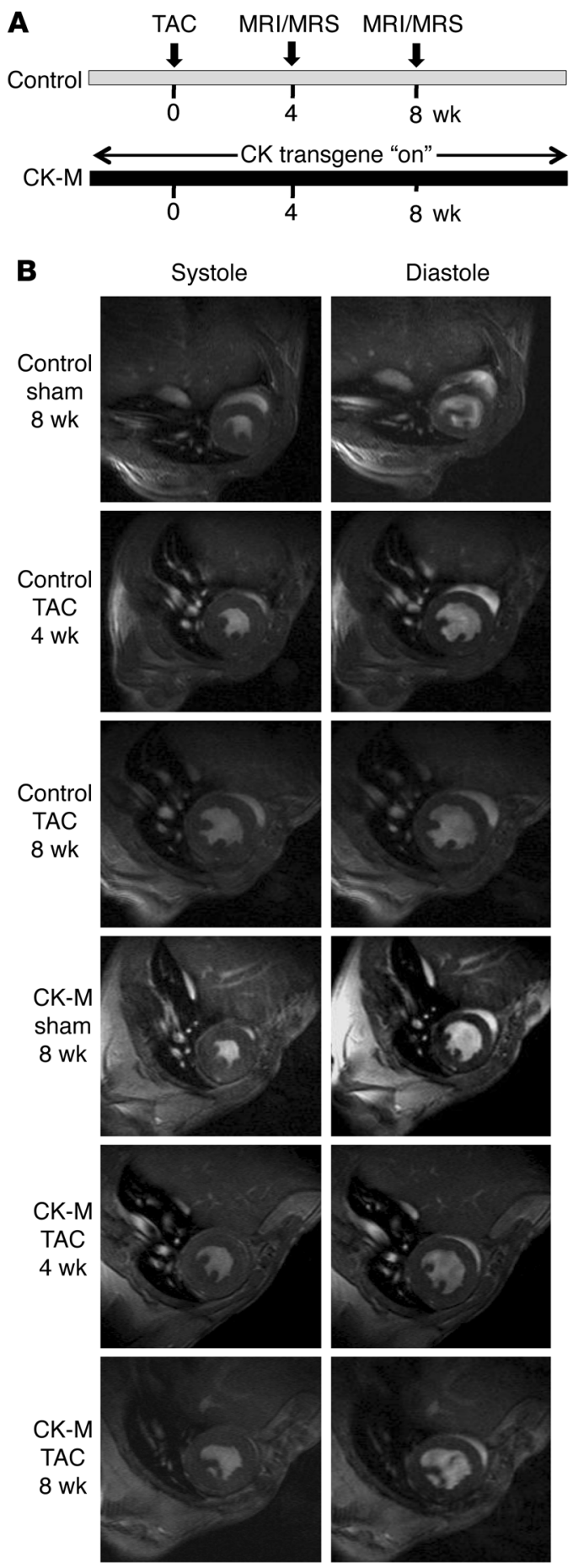

C
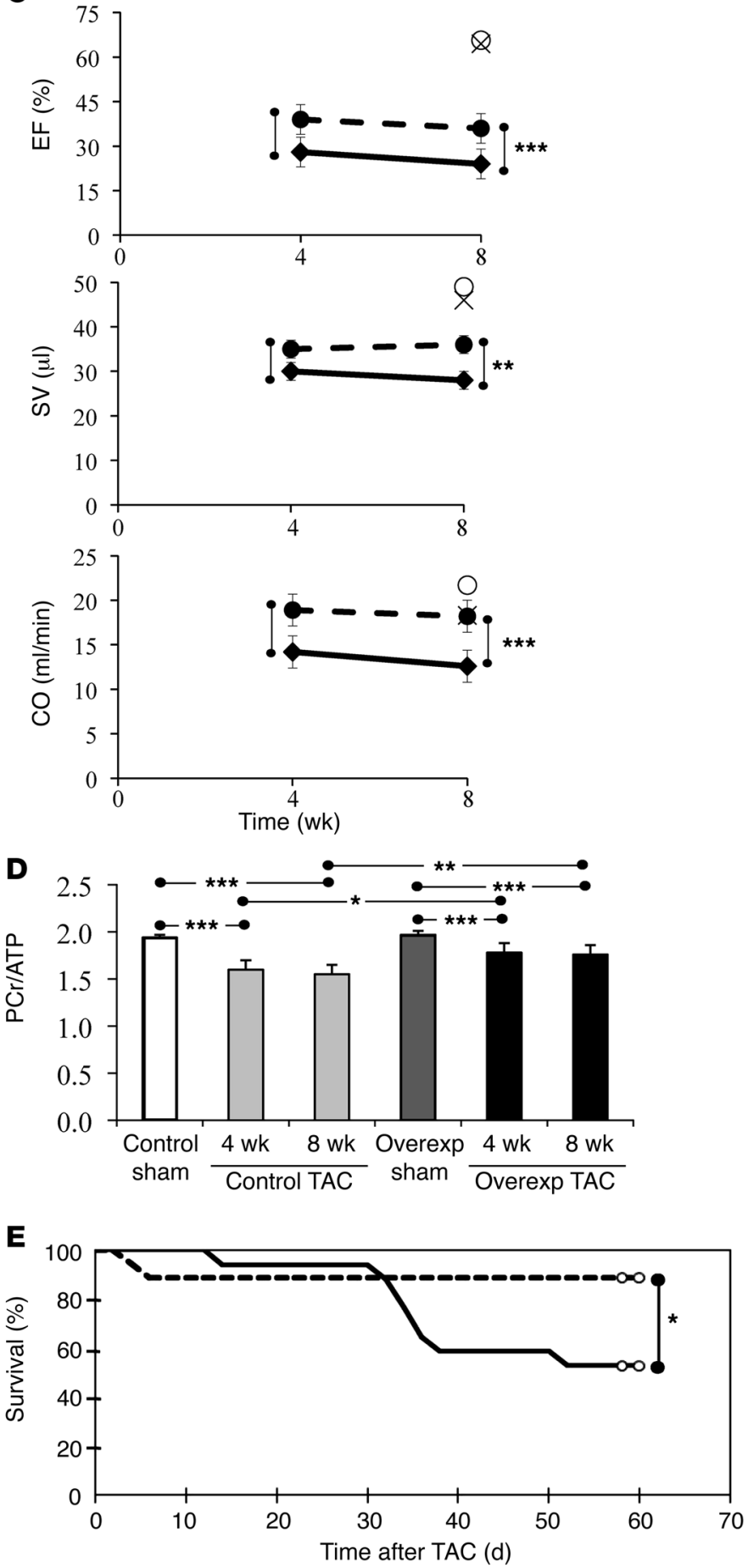

Figure 5

Functional and survival effects of CK-M overexpression in TAC hearts. (A) Time course of experimental protocol and (B) typical transverse in vivo ${ }^{1} \mathrm{H}$ MR images of the mid-LV at end-systole and end-diastole after 8 weeks of sham or TAC surgery in control and CK-M mice. (C) Summary $\mathrm{MRI}$ findings (mean $\pm \mathrm{SD}$ ) at 4 and 8 weeks showing EF, SV, and CO in CK-M sham (open circles, $n=8)$ and control sham (X, $n=9)$ mice, as well as in CK-M TAC (filled circles, dashed lines, $n=8$ ) and control TAC mice (filled diamonds, solid lines, $n=13$ ). (D) In vivo cardiac PCr/ATP ratio in control sham $(n=9)$, control with TAC ( $n=13$ at 4 weeks, $n=9$ at 8 weeks), CK-M overexpressor (Overexp) sham ( $n=8)$, and CK-M with TAC (Overexp TAC; $n=8$ at 4 weeks, $n=7$ at 8 weeks) mouse hearts. (E) Kaplan-Meier survival curve showing improved survival following TAC in CK-M mice (dotted line $n=18$ ) as compared with control mice (solid line, $n=17$ ). ${ }^{*} P<0.05,{ }^{* *} P<0.001,{ }^{* * *} P<0.0001$. 
A

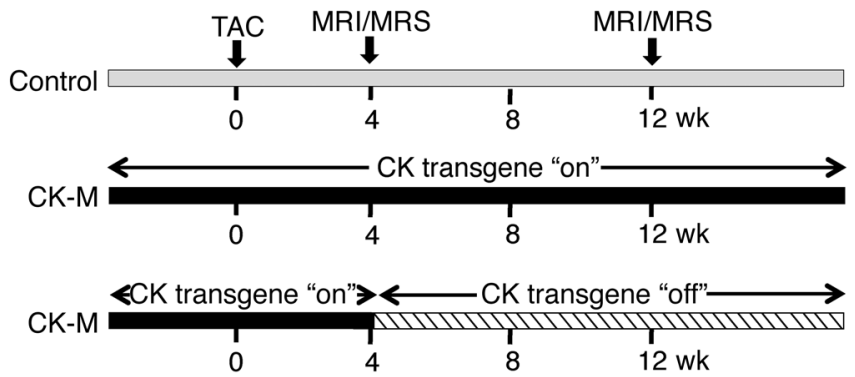

B
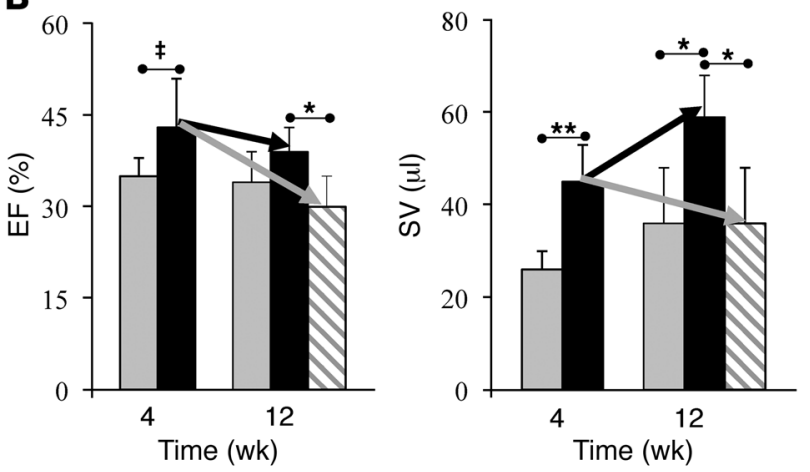

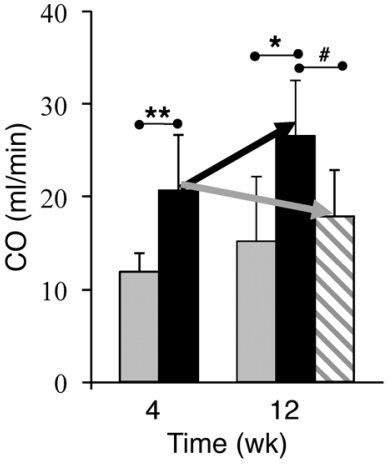

Figure 6

Reversible functional effects of CK-M overexpression in TAC hearts. (A) Time course of experimental protocol and (B) summary of in vivo cardiac functional parameters (mean $\pm S D$ ) derived from $\mathrm{MRI}$; EF, SV, and CO in control animals (gray bars), CK-M animals with the CK-M transgene turned "on" during the entire experiment (black bars), and CK-M animals with the CK-M transgene turned "off" after 4 weeks of TAC (crosshatched bars). $n=5-10$ animals in each group. ${ }^{*} P<0.05,{ }^{\ddagger} P<0.01,{ }^{\#} P=0.07$, ${ }^{* *} P<0.001$. another consequence of, the anatomic remodeling and mechanical dysfunction associated with heart failure. Here we show that CK-M overexpression in the failing heart significantly increases the rate of in vivo ATP delivery, enhances systolic function and CO at baseline and in response to adrenergic stress, and improves survival. Although CK-M overexpression does not limit remodeling in the failing $\mathrm{LV}$, the improvement in in vivo contractile function in $\mathrm{HF}$ is reversible and dependent on persistent CK-M overexpression.

The $\mathrm{CK}$ reaction is a rapid, reversible reaction that transfers a high-energy phosphoryl group between $\mathrm{PCr}$ and ATP, providing an energy reservoir for cells with high, time-varying energy demands, such as spermatozoa, and those of the brain and of skeletal and cardiac muscle (6). Because there are different mitochondrial and cytosolic isoforms of the CK enzyme, it has also been postulated that CK acts as a spatial ATP buffer, enhancing transfer of highenergy phosphates from the mitochondrial sites of generation to cytosolic sites of utilization, including the myofibrils $(6,25)$. There is evidence that $\mathrm{CK}$ is part of a spatially arranged enzymatic network participating in efficient intracellular energy transfer and signaling (26). In the normal heart, an approximately $75 \%$ increase in cardiac CK-M expression increases in vitro total CK activity by about $70 \%$ (Figure 1) and the in vivo ATP synthesis rate through CK by approximately $50 \%$ (Figure $8 \mathrm{I}$ ). CK-M cardiac overexpression in the nonfailing heart does not, however, affect the relative or absolute concentrations of myocardial high-energy phosphates (Figure 4A, Figure 5D, and Figure 8, E-G), baseline indices of contractile function (Figure 5C and Supplemental Figure 3), or the response to adrenergic stimulation (Figure 7 and Supplemental Figure 3). These first observations of CK-M overexpression indicate that the $\mathrm{CK}$ reaction is not limiting energy transfer at baseline or during stress in the normal, nonfailing heart.

The role of CK in HF was questioned in recent years when transgenic CK-null mice constitutively lacking the M-isoform (CK-M), the mitochondrial-isoform (CK-mito), or both were created and found not to develop overt heart failure (13). Two more recent lines of evidence explain this apparent discrepancy. First, contractile function in CK-null mice is not normal, since the in vivo mechanical response to adrenergic stimulation is impaired (i.e., "reduced contractile reserve") (27). Second, CK is only one of several highenergy phosphoryl transfer pathways in tissues with time-varying energy demands that can serve as temporal or spatial high-energy phosphate buffers (26). In muscle of constitutive CK-null mice, phosphoryl transfer through other pathways including adenylate kinase and hexokinase is increased (28), and cytoarchitectural remodeling occurs to more closely approximate energy production and utilization sites (29). In HF, adenylate kinase activity is increased, and this partially offsets the CK-mediated loss of ATPbuffering capacity (30). Thus, adaptive increases in other energy buffering reactions provide some energy reserve in constitutive CK-null mice but are insufficient to fuel normal in vivo contractile function. These first studies showing that augmented CK-M expression improves in vivo metabolism and contractile function in dysfunctional hearts demonstrate that impaired CK metabolism limits function, at least in part, in the failing heart and therefore that the failing heart is "energy starved" as it relates to CK.

Exercise intolerance occurs early in HF, limits functional capacity, and significantly impacts quality of life. Therefore, studies of the metabolic and functional response to adrenergic stress are a compelling aspect of these observations, especially since CK is the major myocardial energy reserve reaction. CK-M overexpression did not significantly augment contractile reserve in normal hearts (Figure 7, C and E, and Supplemental Figure 3). However, in the failing mouse heart, CK-M overexpression increased the response to adrenergic stimulation (i.e., greater increase in $\mathrm{EF}$ and decrease in ESV; Figure 7, D and F) and enabled attainment of significantly higher contractile parameters during stress, including $\mathrm{CO}$ (Results and Supplemental Figure 4, G-J). In healthy hearts (31), including those of mice (23) and humans (32), high-energy phosphates are generally maintained during exercise or adrenergic stimulation, except possibly for modest changes at maximal stimulation 
A

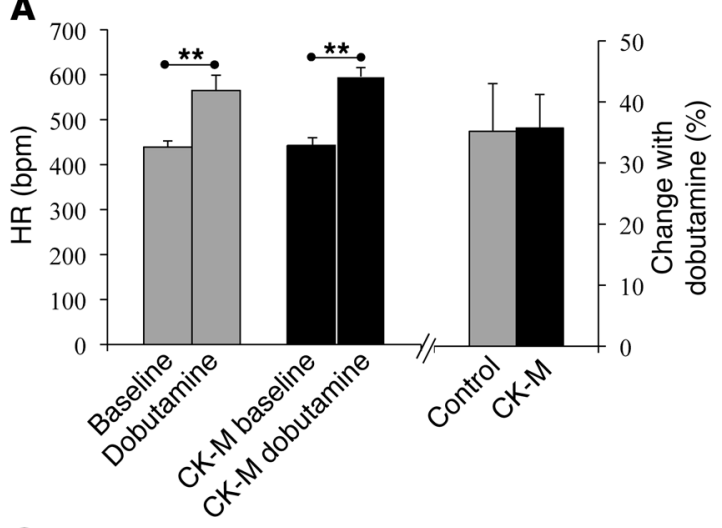

C

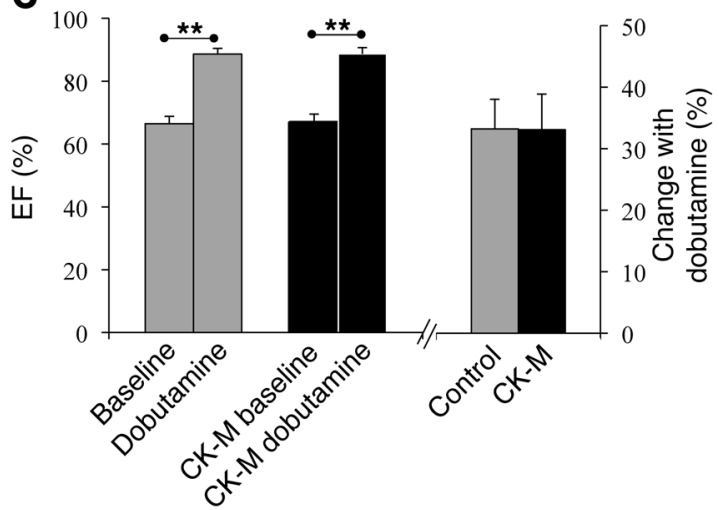

$\mathbf{E}$

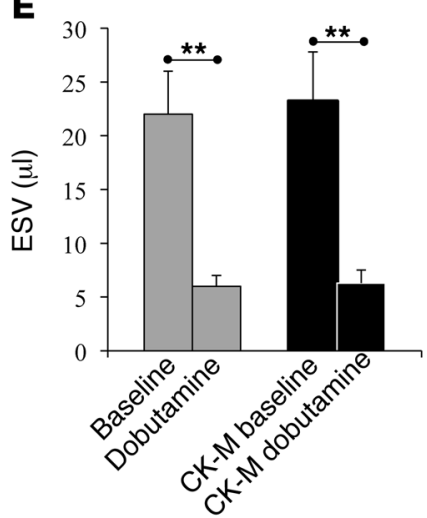

$\mathbf{B}$

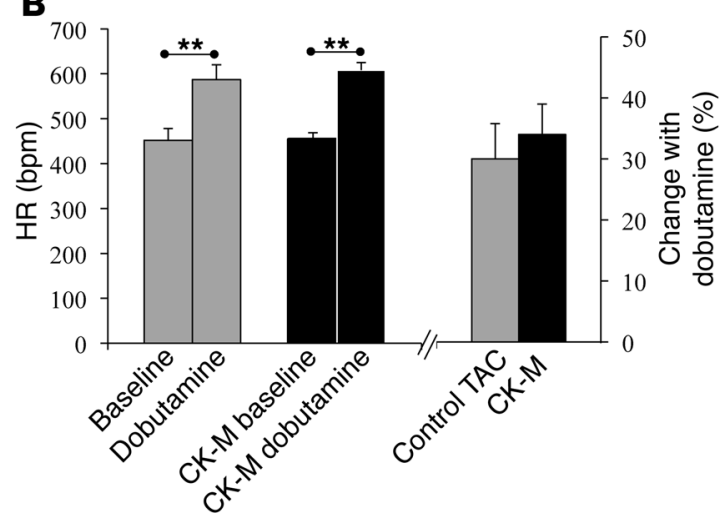

D
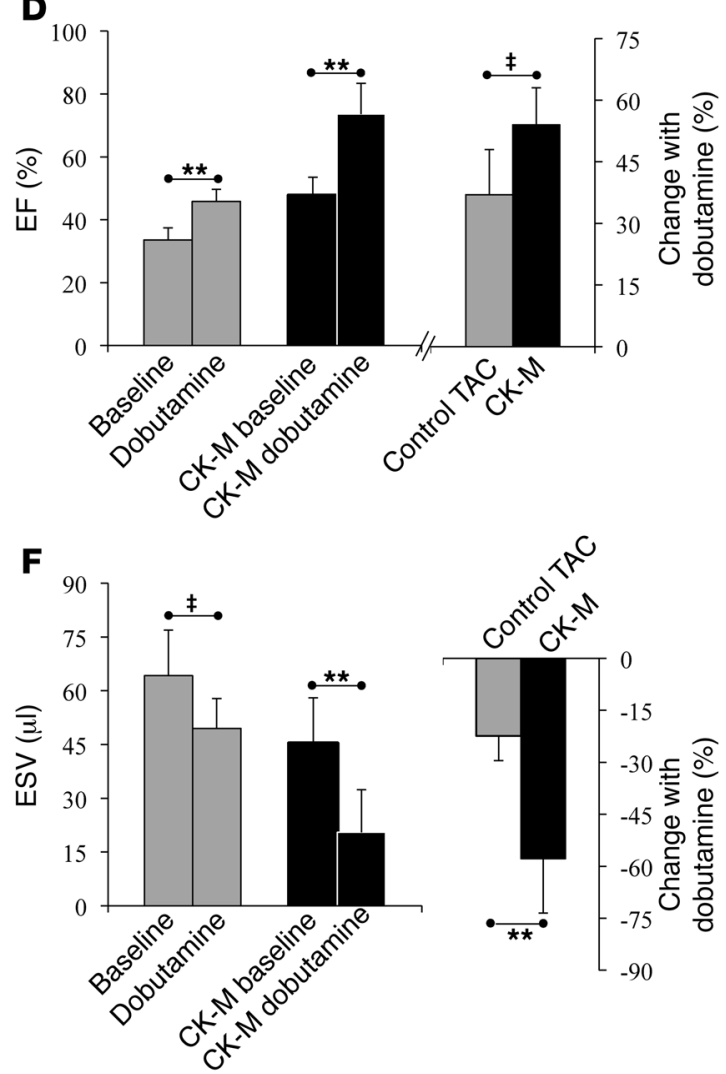

Figure 7

Functional response to adrenergic stress. Effects of dobutamine stress on heart rate (HR; $\mathbf{A}$ and $\mathbf{B}), E F(\mathbf{C}$ and $\mathbf{D})$, and LV ESV (E and $\mathbf{F})$ in sham (A, C, and E) and TAC animals (B, D, and F) in both control (gray bars) and CK-M "on" (black bars) animals ( $n=6-10$ in each group). The change induced by dobutamine (as percentage of baseline values) is shown in the right plot of each panel. Note that although the contractile response of control and CK-M overexpressing animals was similar under sham conditions (A, C, and E), the dobutamine-induced changes in mean EF and ESV were significantly greater in CK-M TAC hearts than in control TAC hearts (B, $\mathbf{D}$, and $\mathbf{F})$. Results are mean $\pm \mathrm{SD}$. ${ }^{\ddagger} P<0.005,{ }^{\star \star} P<0.001$.

$(33,34)$. Data are very limited on myocardial high-energy phosphates during stress in failing human hearts, but there is anecdotal evidence for high-energy phosphate decline during adrenergic stress-induced heart failure decompensation (35). The higher level of cardiac output and function attained during dobutamine stress in failing CK-M TAC hearts was accomplished with an unchanged, and nearly normal, cardiac PCr/ATP ratio, suggesting that increased contractile performance during acute stress does not exact an adverse energetic toll in CK-M failing hearts.
Although cardiac-specific CK-M overexpression reversibly improved contractile function in the failing heart (Figure 6), it did not completely normalize contractile function, nor did it attenuate LV remodeling. It is possible that overexpression of the mitochondrial CK isoform, or even of the other cytosolic CK isoform (CK-B), might have a greater impact on myocardial dysfunction, given that HF-induced reductions in the mitochondrial isoform are closely related to ATP flux through CK (36) and that CK-B expression increases in heart failure (9), a presumably adaptive response. 


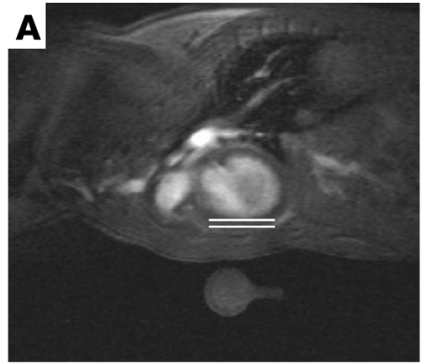

D
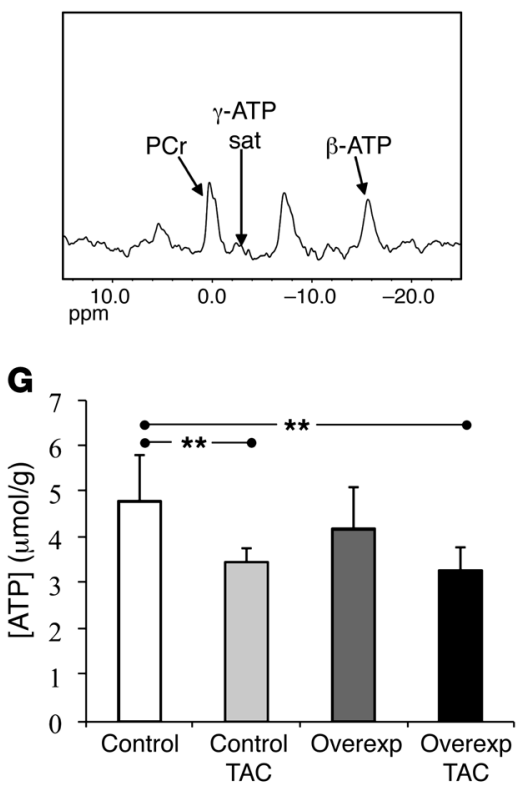

B

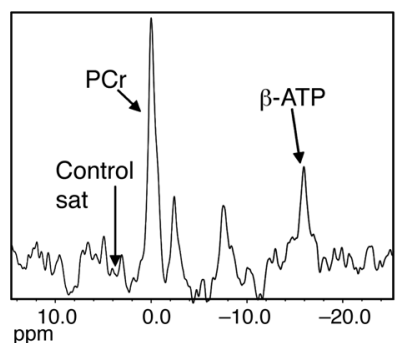

E

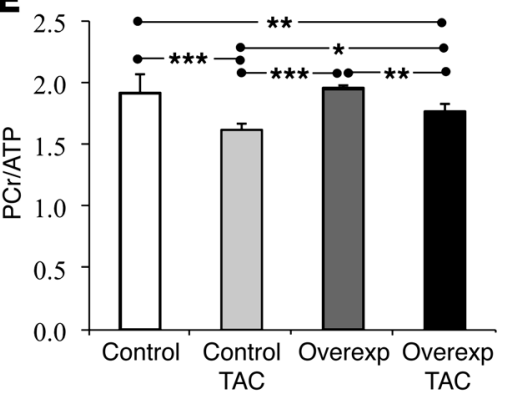

H

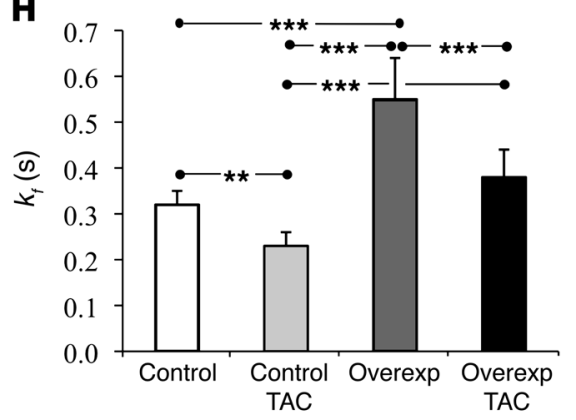

C

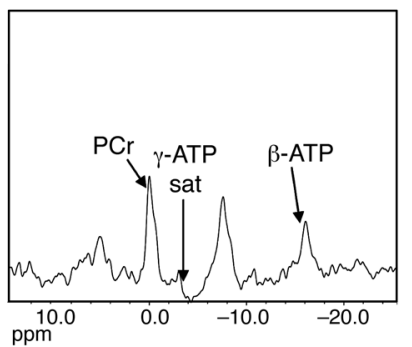

$\mathbf{F}$

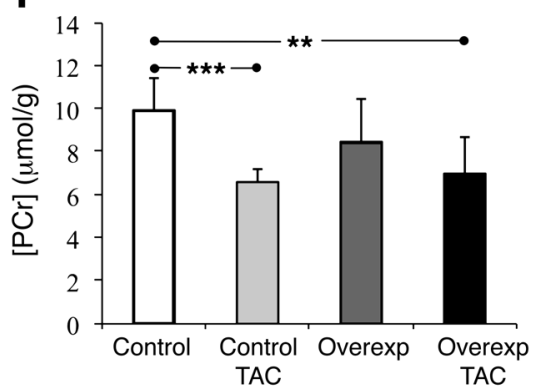

I

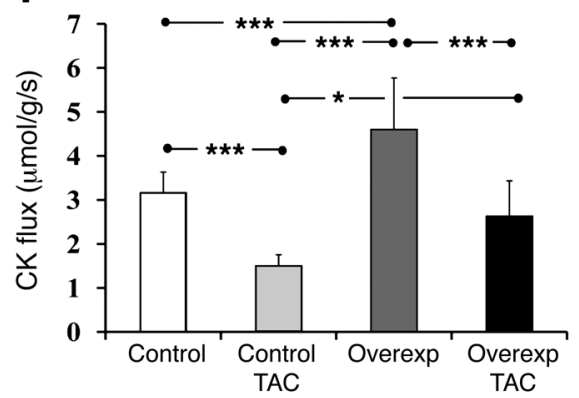

Figure 8

In vivo determination of ATP synthesis rates through CK in the mouse heart. (A) Typical transverse ${ }^{1} \mathrm{H}$ MR image of a mouse at the mid-LV with the nominal location of ${ }^{31} \mathrm{P}$ MR cardiac voxel denoted between the white lines. (B) ${ }^{31} \mathrm{P}$ MR spectrum with control saturation and TR $=10$ seconds and NEX $=16$. (C) Spectrum with $\gamma$-phosphate of ATP saturated with TR $=6$ seconds, NEX $=32$. (D) Spectrum with $\gamma$-phosphate of ATP saturation with TR $=1.5$ seconds, NEX $=96$. $\beta$-ATP; $\beta$-phosphate of ATP. (E-I) Summary of in vivo energetics (mean + SD) for control no-TAC (Control, $n=11$ ), control with TAC (Control TAC, $n=10$ ), CK-M overexpressors no-TAC (Overexp, $n=8$ ), and CK-M overexpressors with TAC (Overexp TAC, $n=7$ ) mice. (E) PCr/ATP ratio. (F) PCr concentration ( $\mu \mathrm{mol} / \mathrm{g}$ wet weight). (G) ATP concentration ( $\mu \mathrm{mol} / \mathrm{g}$ wet weight). (H) CK forward pseudo-first-order rate constant $\left(k_{f}, \mathrm{~S}^{-1}\right)$. (I) rate of ATP synthesis through CK (CK flux, $\left.\mu \mathrm{mol} / \mathrm{g} / \mathrm{s}\right)$. Some of the data in control mice, but not CK-M overexpressers, were previously reported (12). ${ }^{*} P<0.05,{ }^{* *} P<0.01,{ }^{* * \star} P<0.001$.

Nevertheless, CK-M is the predominant isoform in the adult mammalian heart and, as such, was a logical first target, whose overexpression indeed improved ATP synthesis and contractile function in the failing mouse heart. It is clear that TAC-induced HF significantly reduced native $\mathrm{CK}$ capacity and that attributable to the overexpressed CK-M transgene, as reflected by ATP flux through CK (Figure 4 and Figure 8I). Thus, this genetic approach can be considered a "brute force" means to augment CK capacity in HF, and future work is needed to identify specific mechanisms that impair $\mathrm{CK}$ in $\mathrm{HF}$, as these would offer a direct, non-genetic approach to preventing and reversing the critical decline in CK capacity in HF.

Contractile function is dependent on energy delivery and calcium handling, which are linked. Energy is required to fuel the calcium pumps that maintain calcium concentrations in a physiologic range. We studied the relationship between calcium and contraction in isolated ventricular myocytes, where TAC had negative inotropic and lusitropic effects but no effect on calcium transient amplitude. CK-M overexpression only further delayed the temporal recovery of the calcium transient and had no impact on sarcomere shortening in isolated myocytes (Figure 9). These observations suggest that CK-M overexpression does not alter fundamental contractile function or maximal calcium release in the isolated myocyte, whose energetic demands while unloaded and stimulated at $0.5 \mathrm{~Hz}$ are more than an order of magnitude below that of the in vivo, hemodynamically loaded mouse heart beating at $10 \mathrm{~Hz}$. This was an important avenue for investigation, because the products of ATP hydrolysis (e.g., ADP + inorganic phossphate [Pi]) inhibit calcium pump activity and adversely affect the relationship between calcium and the myofilaments $(37,38)$. Indeed, the reduction in PCr in TAC hearts could be associated with an increase in Pi that cannot be quantified in vivo. Nevertheless, we did not observe any changes in sarcomere shortening or calcium transient amplitude in CK-M-overexpressing myocytes, keeping in mind that augmentation of CK energy reserve capacity likely has a greater impact in settings with high energy demand (i.e., the chronically failing in vivo heart) than those with low energy requirements (i.e., unloaded, slowly contracting isolated myocytes). 


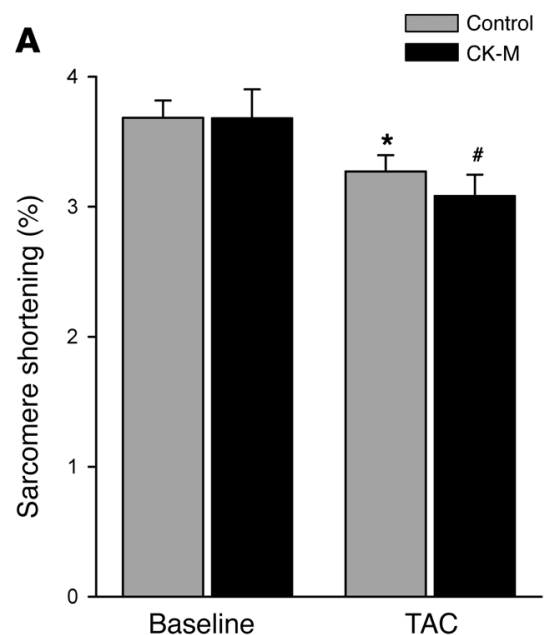

C

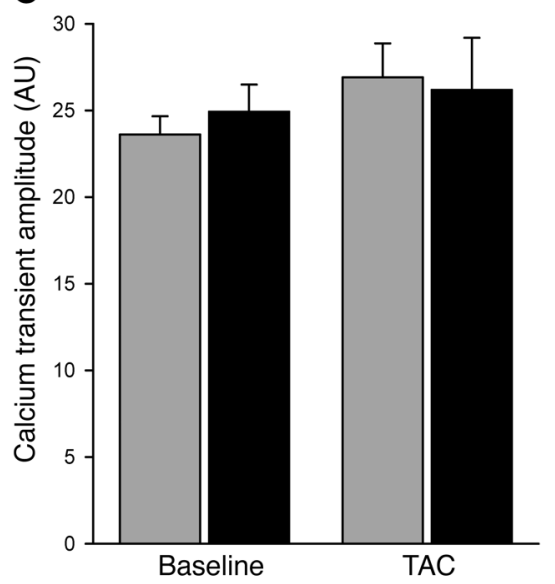

B

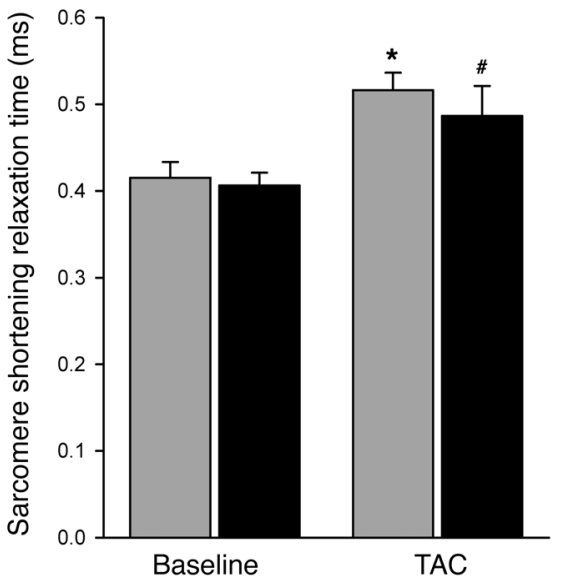

D

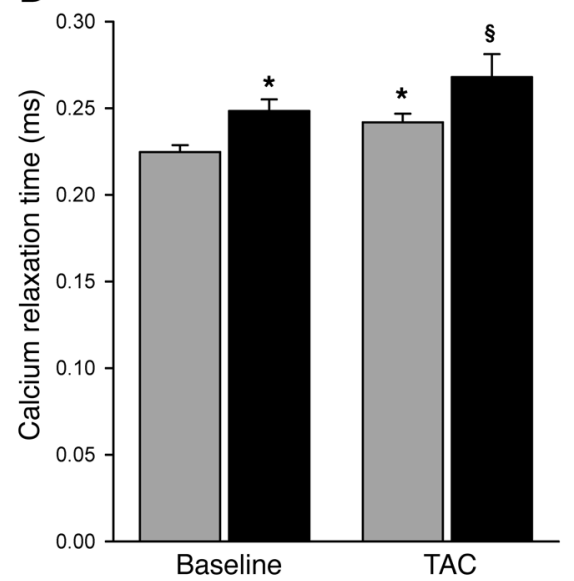

skeletal alterations in HF, including actin depolymerization and the modification of desmin by advanced glycation end products (44). Thus, CK-M overexpression may have many effects in HF beyond an increase in $\mathrm{PCr} / \mathrm{ATP}$ ratio.

It is important to emphasize that these translational studies derive directly from clinical observations. Hypertension affects as many as $60 \%$ of older individuals in industrialized societies and often results in pressure-overload hypertrophy, and eventually HF and increased cardiovascular mortality (45). Because the kinetics of ATP flux through CK, rather than CK pool sizes, differ between failing and nonfailing human pressure-overload hypertrophied hearts (15), we chose here a gene therapy strategy designed to augment CK enzyme activity, rather than CK pool sizes. A recent transgenic strategy that increased $\mathrm{Cr}$ and $\mathrm{PCr}$ pools did not improve murine cardiac contractile function, but instead attenuated it (46). In contrast, our strategy of augmenting CK expression, activity, and in vivo ATP synthesis through CK improved LV function in failing hearts. These observations directly demonstrate that metabolic augmentation of the rate of myofibrillar ATP delivery offers an attractive opportunity to improve contractile function and important clinical outcomes in HF.

\section{Methods}

Animal studies. All transgenic mouse development work was performed at the transgenic core facility of UCLA. Transgenic mice were developed on the basis of the Tet-off system (47). At the first stage, two types of mice were generated: first, those with a transgene for the regulatory

Work more than 15 years ago $(20,21,39)$ suggested that the normal heart has considerable excess CK capacity in that loss of approximately $90 \%$ of CK activity had minor effects on baseline systolic function, although this was not the case in all studies (40). However, CK loss impaired contractile reserve and caused diastolic dysfunction $(21,41)$. In the present study, the contractile benefits of CK-M overexpression were observed only in failing hearts both acutely in response to adrenergic stimulation (Figure 7 and Supplemental Figure 4) and chronically in terms of functional (Figure 5C and Figure 6B) and survival improvements (Figure 5E). Such observations are consistent with prior studies in which longerterm CK inhibition ( $>40$ minutes) impaired in vivo function in normal porcine hearts (42) or resulted in $100 \%$ mortality following myocardial infarction in rats (43). Thus, the role of CK is magnified over time and in the failing heart. The increased energetic demand and altered substrate metabolism in HF (5) may be important contributing factors to these observations of improved function with CK-M overexpression in the failing heart, as well as the very recent appreciation that CK-M loss contributes to cyto- protein tTA (tetracycline-controlled transactivator) under the control of the $\alpha$-MHC promoter; and second, mice expressing the CK-M transgene under the control of tetracycline-responsive element (TRE) following microinjection of the CK-M construct into fertilized mouse embryos (C57BL/6 strain). After the crossing of TRE-CK-M mice with $\alpha$-MHCtTA mice, double transgenic mice (CK-M-tTA) were confirmed by genotyping, and CK-M transgene induction was achieved by switching from a diet containing doxycycline (650 mg/kg body weight) to a regular chow diet at least 4 weeks prior to study (designated CK-M overexpressors) and typically shortly after the time of weaning. Control mice were littermates containing either the tTA or CK-M transgene alone or double transgenic CK-M-tTA mice remaining on the doxycycline diet. Six- to 10-week-old male mice underwent sham or TAC surgery as previously described $(17,48)$. Prior studies had shown that this method of TAC results in cardiac dilatation and dysfunction after 3-4 weeks, as well as a reduction in cardiac PCr/ATP ratio and ATP flux through CK of a magnitude similar to that in human heart failure $(12,17,49)$.

${ }^{31} \mathrm{P}$ NMR spectroscopy of perfused hearts. Mice were heparinized (500 U i.p.) and anesthetized with sodium pentobarbital ( $80 \mathrm{mg} / \mathrm{kg}$ body weight). Hearts were isolated and subjected to retrograde perfusion via the aortic 
cannula (Langendorff mode) at a constant temperature of $37^{\circ} \mathrm{C}$ and constant pressure of $100 \mathrm{~cm} \mathrm{H}_{2} \mathrm{O}$ with modified Krebs-Henseleit buffer. The buffer was saturated with $95 \% \mathrm{O}_{2}$ and $5 \% \mathrm{CO}_{2}$ and contained (in $\mathrm{mM}$ ) $118.5 \mathrm{NaCl}, 25 \mathrm{NaHCO}_{3}, 5.9 \mathrm{KCl}, 1.2 \mathrm{MgSO}_{4}, 1.6 \mathrm{CaCl}_{2}, 1 \mathrm{Na}$ pyruvate, and 5 glucose. Perfused hearts were placed into a $10-\mathrm{mm}$ diameter NMR tube and lowered into a Bruker Avance 500 (11.7T) vertical bore MR system interfaced with XWIN-NMR software. The effluent was removed from the NMR tube via vacuum suction, with the liquid level kept well above the heart.

${ }^{31} \mathrm{P}$ MR spectra were collected at $202.5 \mathrm{MHz}$ without proton decoupling by signal averaging 128 scans using a sweep width of $8,090 \mathrm{~Hz}$. Control spectra without pre-saturation (pulse width $12 \mu$ s, flip angle 67 degrees, relaxation delay [d1] 1 second) were obtained immediately before and after the magnetization transfer experiment to assess the stability of the preparation over the course of the experiment. Magnetization transfer was performed (128 scans, pulse width $12 \mu$ s, flip angle 67 degrees) by applying a continuous wave saturation pulse at the $\gamma$-phosphate of ATP for $8 \mathrm{sec}-$ onds and measuring of the PCr signal intensity $\left(\mathrm{M}_{0}{ }^{\prime}\right)$. A control experiment was performed in which the selective irradiation was placed downfield at a frequency equidistant from the $\mathrm{PCr}$ resonance before measuring the $\mathrm{PCr}$ signal intensity $\left(\mathrm{M}_{0}\right)$. The intensity of $\mathrm{PCr}$ resonance decays from $\mathrm{M}_{0}$ to $\mathrm{M}_{0}{ }^{\prime}$ as $\gamma$-ATP is saturated. A complete magnetization transfer experiment was performed in 36 minutes.

To determine the apparent relaxation time $\left(\mathrm{T} 1^{\prime}\right)$ of $\mathrm{PCr}$ (i.e., relaxation time in the presence of saturating irradiation), we acquired ${ }^{31} \mathrm{P}$ spectra by signal averaging 128 scans with d1's of $0.3,0.4,0.7,1.1,1.7,2.8,4.0$, and 6.0 seconds (saturating pulse at $\gamma$-ATP, pulse width $16.5 \mu$ s, flip angle 90 degrees). The different d1's were applied randomly to minimize any effects of experimental time. The exponential increase in the PCr signal intensity with increasing d1's was analyzed using LABFit curve fitting software to determine $\mathrm{T} 1^{\prime}$. Intrinsic $\mathrm{T} 1$ (i.e., relaxation time of $\mathrm{PCr}$ in the absence of chemical exchange) was then calculated using the equation $\mathrm{T} 1=\mathrm{T} 1^{\prime} /$ $\left(\mathrm{M}_{0}{ }^{\prime} / \mathrm{M}_{0}\right)(50)$. The pseudo-first-order rate constant $\left(k_{f}\right)$ for the forward CK reaction $\left(\mathrm{PCr}+\mathrm{ADP}+\mathrm{H}^{+} \leftrightarrow \mathrm{ATP}+\mathrm{Cr}\right)$ was then calculated as $k_{f}=\left(\mathrm{M}_{0}-\mathrm{M}_{0}{ }^{\prime}\right) /$ $\left(\mathrm{T} 1 \times \mathrm{M}_{0}{ }^{\prime}\right)$ as described previously (51). Subsequently, flux through the CK reaction (i.e., rate of ATP synthesis from $\mathrm{PCr}$ ) was calculated as the product of $k_{f}$ and $[\mathrm{PCr}](14,15)$.

In vivo MRI/MRS studies. In vivo MRI/MRS experiments were performed on a Bruker spectrometer equipped with a 4.7-T/40-cm Oxford magnet and actively shielded gradients. The studies were conducted with an in vivo cardiac combined MRI/MRS protocol as previously described (17-19). A complete set of high temporal and spatial resolution multi-slice cine MR images was acquired of the entire LV without gaps to assess $L V$ mass, ventricular volumes, and $\operatorname{EF}(18,19)$. After imaging and without repositioning the animal, a one-dimensional ${ }^{31} \mathrm{P}$ chemical shift imaging (1D-CSI) sequence was used to obtain high-energy phosphate data. The PCr and $[\beta-P]$ ATP peaks in ${ }^{31} \mathrm{P}$ MR localized spectra were quantified by integration of the peak areas (18) by investigators blinded to the group assignment. The technique of voxel shifting (52) permitted us to redefine the slice boundaries fractionally during processing after data collection in order to align them with the LV border using the high-resolution ${ }^{1} \mathrm{H}$ image as a guide and thus minimize contamination from the chest wall. After the mouse exams were completed, external phantom experiments were also performed on the same day with the same coil set under comparable conditions in order to calculate the absolute concentrations of PCr and ATP, as described previously (49).

To measure the rate of ATP synthesis through cardiac CK, a separate triple repetition time saturation transfer (TRiST) MRS study was performed in some animals using a previously published protocol $(12,53)$. Specifically, localized ${ }^{31} \mathrm{P}$ MR spectra were obtained with a 1D-CSI sequence $(16 \mathrm{~mm}$ field of view, 16 phase encoding steps) using modified BIR $490^{\circ}$ adiabatic pulses. Two acquisitions with different repetition periods (repetition time
$[\mathrm{TR}]=1.5 \mathrm{~s}$, number of excitations $[\mathrm{NEX}]=96$ and $\mathrm{TR}=6 \mathrm{~s}, \mathrm{NEX}=32$ ) were acquired in the presence of a saturating irradiation pulse applied to the exchanging CK moiety, namely, $\gamma$-phosphate of ATP at $-2.5 \mathrm{ppm}$, relative to $\mathrm{PCr}$, and another acquisition, fully relaxed $(\mathrm{TR}=10 \mathrm{~s}, \mathrm{NEX}=16)$ in the presence of control irradiation applied at $+2.5 \mathrm{ppm}$. The measured signal intensities were normalized for different NEX values. PCr concentration was measured as described previously (49).

To determine the effect of CK-M overexpression on the inotropic, chronotropic, and metabolic response of the normal and failing heart to adrenergic stress, we performed MRI and MRS examinations at baseline and after i.p. bolus injection of dobutamine $(1.5 \mu \mathrm{g} / \mathrm{g}$ body weight $)(22,23)$ in the absence and presence of TAC in control and CK-M-overexpressing mice.

Biochemistry. CK protein expression was determined by standard Western blotting techniques as previously reported (54-56). LV homogenates were separated on a $4 \%-12 \%$ Bis-Tris gradient gel under denaturing conditions as previously described $(55,56)$ and using an anti-CK-M $166-180$ antibody produced in rabbit IgG fraction of antiserum and electroblotted onto nitrocellulose membrane $(55,56)$. Primary antibody binding was visualized using a horseradish peroxidase-based chemiluminescence assay (Figures 1 and 2 and Supplemental Figure 5).

Heart tissues were powdered in liquid $\mathrm{N}_{2}$ with mortar and pestle and extracted with $150 \mathrm{mmol} / \mathrm{l} \mathrm{NaCl}, 60 \mathrm{mmol} / \mathrm{l}$ Tris- $\mathrm{HCl}$ ( $\mathrm{pH} 7.5$ ), $5 \mathrm{mmol} / \mathrm{l}$ EDTA, $0.2 \%$ Triton X-100, $1 \mathrm{mmol} / \mathrm{l} \mathrm{PMSF}, 10 \mathrm{mg} / \mathrm{ml} \mathrm{leupeptin,} \mathrm{and} 1 \mathrm{mg} / \mathrm{ml}$ aprotinin. Extracts were centrifuged $\left(10\right.$ minutes, $\left.10,000 \mathrm{~g}, 4^{\circ} \mathrm{C}\right)$, and adenylate kinase and hexokinase enzyme activities were measured with coupled enzyme assays with a SpectraMax M2 spectrophotometer (Molecular Devices) at $340 \mathrm{~nm}$, as previously described (30). Total CK activity was also measured in homogenized heart tissue from the same animals as previously described (12).

Isolated myocyte studies. Sarcomere shortening, whole $\mathrm{Ca}^{2+}$ transient, and their kinetics were assayed in freshly isolated myocytes as previously described (57).

Statistics. Results are presented as mean \pm SD. Survival curves were generated using the Kaplan-Meier survival function using XLSTAT statistical software (Microsoft). Data were tested for normality, and parametric testing was used when appropriate. Comparisons between 2 groups were performed with the paired or unpaired Student's $t$ test, as appropriate. Comparisons among multiple groups were performed with 1-way ANOVA, and if a significant difference was observed, pairwise comparisons were performed with the Tukey-Kramer comparisons test. To adjust for repeated measures in the same animal (Figure 5C), a generalized estimating equation (GEE) model was used to adjust for repeated measures.

Study approval. Studies were approved by the Institutional Animal Care and Use Committee of the Johns Hopkins University.

\section{Acknowledgments}

This work was funded by NIH grants HL63030 and HL61912, a grant from the Mid-Atlantic affiliate of the American Heart Association, and support from the Donald W. Reynolds Foundation and the Clarence Doodeman Endowment. We would like to thank Haiying Pu and Jing Gao for their technical assistance in generating the $\mathrm{CK}$ transgenic mice.

Received for publication February 4, 2011, and accepted in revised form November 2, 2011.

Address correspondence to: Robert G. Weiss, Carnegie 584, The Johns Hopkins Hospital, 600 N. Wolfe St., Baltimore, Maryland 21287-6568, USA. Phone: 410.955.1703; Fax: 410.614.9398; E-mail: rweiss@jhmi.edu. 
1. Pfeffer MA, et al. Myocardial infarct size and ventricular function in rats. Circ Res. 1979;44(4):503-512.

2. Hunt SA, et al. 2009 focused update incorporated into the ACC/AHA 2005 Guidelines for the Diagnosis and Management of Heart Failure in Adults: a report of the American College of Cardiology Foundation/American Heart Association Task Force on Practice Guidelines: developed in collaboration with the International Society for Heart and Lung Transplantation. Circulation. 2009;119(14):e391-e479.

3. IngwallJS. Is cardiac failure a consequence of decreased energy reserve? Circulation. 1993;87(suppl VII): VII-58-VII-62.

4. Katz AM. Is the failing heart energy depleted? Cardiol Clin. 1998;16(4):633-644.

5. Stanley WC, Recchia FA, Lopaschuk GD. Myocardial substrate metabolism in the normal and failing heart. Physiol Rev. 2005;85(3):1093-1129.

6. Wallimann T. Bioenergetics. Dissecting the role of creatine kinase. Curr Biol. 1994;4(1):42-46.

7. Saks VA. Creatine kinase isozymes and the control of cardiac contraction. In: Jacobus WE, Ingwall JS, eds. Heart Creatine Kinase. Baltimore, Maryland, USA: Williams \& Wilkins; 1980:109-124.

8 . Ingwall JS, et al. The creatine kinase system in normal and diseased human myocardium. $N$ Engl J Med. 1985;313(17):1050-1054.

9. Nascimben L, et al. Creatine kinase system in failing and nonfailing human myocardium. Circulation. 1996;94(8):1894-1901

10. Hardy CJ, Weiss RG, Bottomley PA, Gerstenblith G. Altered myocardial high-energy phosphate metabolites in patients with dilated cardiomyopathy. Am Heart J. 1991;122(3 pt 1):795-801.

11. Ye Y, et al. Myocardial creatine kinase kinetics and isoform expression in hearts with severe LVH hypertrophy. Am J Physiol Heart Circ Physiol. 2001; 281(1):H376-H386.

12. Gupta A, Chacko VP, Schär M, Akki A, Weiss RG. Impaired ATP kinetics in the in vivo failing mouse hearts. Circ Cardiovasc Imaging. 2011;4(1):42-50

13. Saupe KW, Spindler M, Tian R, Ingwall JS. Impaired cardiac energetics in mice lacking muscle specific isoenzymes of creatine kinase. Circ Res. 1998; 82(8):898-907.

14. Weiss RG, Gerstenblith G, Bottomley PA. ATP flux through creatine kinase in the normal, stressed, and failing human heart. Proc Natl Acad Sci U S A. 2005; 102(3):808-813.

15. Smith CS, Bottomley PA, Gerstenblith G, Schulman SP, Weiss RG. Altered creatine kinase ATP kinetics in failing hypertrophied human myocardium. Circulation. 2006;114(11):1151-1158.

16. Takimoto E, et al. Chronic inhibition of cyclic GMP phosphodiesterase $5 \mathrm{~A}$ prevents and reverses cardiac hypertrophy. Nat Med. 2005;11(2):214-222.

17. Maslov M, et al. Altered high-energy phosphate metabolism predicts contractile dysfunction and subsequent ventricular remodeling in pressureoverload hypertrophy mice. Am J Physiol Heart Circ Physiol. 2007;292(1):H387-H392.

18. Chacko VP, Aresta F, Chacko SM, Weiss RG. MRI/ MRS assessment of in vivo murine cardiac metabolism, morphology, and function at physiological heart rates. Am J Physiol Heart Circ Physiol. 2000 279(5):H2218-H2224.

19. Weiss RG, et al. An increase in the myocardial $\mathrm{PCr}$ /ATP ratio in GLUT4 null mice. FASEB J. 2002 16(6):613-615.

20. Hamman BL, et al. Inhibition of the creatine kinase reaction decreases the contractile reserve of isolated rat hearts. Am J Physiol. 1995;269(3 pt 2):H1030-H1036.

21. Tian R, Ingwall JS. Energetic basis for reduced contractile reserve in isolated rat hearts. Am JPhysiol. 1996; 270(4 pt 2):H1207-H1216.
22. Wiesmann F, et al. Dobutamine stress magnetic resonance microimaging in mice: acute changes of cardiac geometry and function in normal and failing murine hearts. Circ Res. 2001;88(6):563-569.

23. Naumova AV, Weiss RG, Chacko VP. Regulation of murine myocardial energy metabolism during adrenergic stress studied by in vivo 31P NMR spectroscopy. Am J Physiol Heart Circ Physiol. 2003; 285(5):H1976-H1979.

24. Ingwall JS, Weiss RG. Is the failing heart energy starved? On using chemical energy to support cardiac function. Circ Res. 2004;95(2):135-145

25. Bessman SP, Carpenter CL. The creatine-creatine phosphate energy shuttle. Ann Rev Biochem. 1985; 54:831-862.

26. Dzeja PP, Terzic A. Phosphotransfer networks and cellular energetics. J Exp Biol. 2003;206(pt 12):2039-2047.

27. Crozatzier B, et al. Role of creatine kinase in cardiac excitation-contraction coupling: studies in creatine kinase-deficient mice. FASEB J. 2002;16(7):653-660.

28. Dzeja PP, Terzic A, Wieringa B. Phosphotransfer dynamics in skeletal muscle from creatine kinase genedeleted mice. Mol Cell Biochem. 2004;256(1-2):13-27.

29. Kaasik A, Veksler V, Boehm E, Novotova M, Minajeva A, Ventura-Clapier R. Energetic crosstalk between organelles: architectural integration of energy production and utilization. Circ Res. 2001; 89(2):153-159.

30. Dzeja PP, Vitkevicius KT, Redfield MM, Burnett JC, Terzic A. Adenylate kinase-catalyzed phosphotransfer in the myocardium: increased contribution in heart failure. Circ Res. 1999;84(10):1137-1143.

31. Balaban RS, Kantor HL, Katz LA, Briggs RW. Relation between work and phosphate metabolites in the in vivo paced mammalian heart. Science. 1986; 232(4754):1121-1123.

32. Weiss RG, Bottomley PA, Hardy CJ, Gerstenblith G. Regional myocardial metabolism of high-energy phosphates during isometric exercise in patients with coronary artery disease. $N$ Engl J Med. 1990; 323(23):1593-1600

33. Lamb HJ, et al. Metabolic response of normal human myocardium to high-dose atropine-dobutamine stress studied by 31P-MRS. Circulation. 1997;96(9):2969-2977.

34. Zhang J, et al. Transmural bioenergetic responses of normal myocardium to high workstates. Am J Physiol. 1995;268(5 pt 2):H1891-H1905.

35. Schaefer S, Schwartz GG, Steinman SK, Meyerhoff DJ, Massie BM, Weiner MW. Metabolic response of the human heart to inotropic stimulation: in vivo phosphorus-31 studies of normal and cardiomyopathic myocardium. Magn Reson Med. 1992; 25(2):260-272.

36. Ye Y, Gong G, Ochiai K, Liu J, Zhang J. High-energy phosphate metabolism and creatine kinase in failing hearts: a new porcine model. Circulation. 2001 103(11):1570-1576.

37. Kentish JC. The effects of inorganic phosphate and creatine phosphate on force production in skinned muscles from rat ventricle. J Physiol. 1986; 370:585-604

38. Maack C, O'Rourke B. Excitation-contraction coupling and mitochondrial energetics. Basic Res Cardiol. 2007;102(5):369-392.

39. Shoubridge EA, Jeffrey FM, Keogh JM, Radda GK, Seymour AL. Creatine kinase kinetics, ATP turnover, and cardiac performance in hearts depleted of creatine with the substrate analogue b-guanidinopropionic acid. Biochim Biophys Acta. 1987; 847(1):25-32.

40. Kapelko VI, et al. The cardiac contractile failure induced by chronic creatine and phosphocreatine deficiency. J Mol Cell Cardiol. 1988;20(6):465-479.

41. Tian R, et al. Role of Mg-ADP in the development of diastolic dysfunction in the intact beating rat heart. J Clin Invest. 1997;99(4):745-751.

42. Xiong Q, et al. ATP production rate via creatine kinase or ATP synthase in vivo: a novel superfast magnetization saturation transfer method. Circ Res. 2011;108(6):653-663.

43. Horn M, Remkes H, Stromer H, Dienesch C, Neubauer S. Chronic phosphocreatine depletion by the creatine analogue beta-guanidinopropionate is associated with increased mortality and loss of ATP in rats after myocardial infarction. Circulation. 2001; 104(15):1844-1849.

44. Diquet $\mathrm{N}$, et al. Muscle creatine kinase deficiency triggers both actin depolymerization and desmin disorganization by advanced glycation end-products in dilated cardiomyopathy. J Biol Chem. 2011; 286(40):35007-35019.

45. Levy D, Garrison RJ, Savage DD, Kannel WB, Castelli WP. Prognostic implications of echocardiographically determined left ventricular mass in the Framingham Heart Study. N Engl J Med. 1990; 322(22):1561-1566

46. Wallis J, et al. Supranormal myocardial creatine and phosphocreatine concentrations lead to cardiac hypertrophy and heart failure: insights from creatine transporter-overexpressing transgenic mice. Circulation. 2005;112(20):3131-3139.

47. Ryding ADS, Sharp MGF, Mullins JJ. Conditional transgenic technologies. J Endocrinol. 2001; 171(1):1-14.

48. Takimoto E, et al. cGMP catabolism by phosphodiesterase $5 \mathrm{~A}$ regulates cardiac adrenergic stimulation by NOS3-dependent mechanism. Circ Res. 2005; 96(1):100-109.

49. Gupta A, Chacko VP, Weiss RG. Abnormal energetics and ATP depletion in pressure-overload mouse hearts: in vivo high-energy phosphate concentration measures by noninvasive magnetic resonance. Am J Physiol Heart Circ Physiol. 2009;297(1):H59-H64.

50. Bittl JA, Ingwall JS. Reaction rates of creatine kinase and ATP synthesis in the isolated rat heart: a 31P NMR magnetization transfer study. JBiol Chem. 1985; 260(6):3512-3517.

51. Forsén S, Hoffman RA. Study of moderately rapid chemical exchange reactions by means of nuclear magnetic double resonance. J Chem Phys. 1963; 39(11):2892-2901.

52. Bottomley PA, Hardy CJ, Roemer PB, Weiss RG. Problems and expediencies in human ${ }^{31} \mathrm{P}$ spectroscopy: the definition of localized volumes, dealing with saturation and the technique-dependence of quantification. NMR Biomed. 1989;2(5-6):284-289.

53. Schär M, El-Sharkawy A-M, Weiss RG, Bottomley PA. Triple repetition time saturation transfer (TRiST) 31P spectroscopy for measuring human creatine kinase reaction kinetics. Magn Reson Med. 2010;63(6):1493-1501.

54. Saupe KW, Spindler M, Hopkins JAC, Shen W, Ingwall JS. Kinetic, thermodynamic, and developmental consequences of deleting creatine kinase isoenzymes from the heart. J Biol Chem. 2000; 275(26):19742-19746

55. Schlattner U, Mockli N, Speer O, Werner S, Wallimann T. Creatine kinase and creatine transporter in normal, wounded, and diseased skin. J Invest Dermatol. 2002;118(3):416-423.

56. Wallimann T, Wegmann G, Moser H, Huber R, Eppenberger HM. High content of creatine kinase in chicken retina: compartmentalized localization of creatine kinase isoenzymes in photoreceptor cells. Proc Natl Acad Sci US A. 1986;83(11):3816-3819.

57. Tocchetti CG, et al. Nitroxyl improves cellular heart function by directly enhancing cardiac sarcoplasmic reticulum Ca2+ cycling. Circ Res. 2007; 100(1):96-104. 